\title{
ON GENERALIZED QUATERNION ALGEBRAS
}

\author{
GEORGE SZETO \\ Department of Mathematics \\ Bradley University \\ Peoria, Illinois 61625 \\ U.S.A.
}

(Received February 1, 1979 and in revised form July 13, 1979)

ABSTRACT. Let $B$ be a commutative ring with 1 , and $G(=\{\sigma\})$ an automorphism group of $B$ of order 2. The generalized quaternion ring extension $B[j]$ over B is defined by S. Parimala and R. Sridharan such that (1) $B[j]$ is a free B-module with a basis $\{1, j\}$, and (2) $j^{2}=-1$ and $j b=\sigma(b) j$ for each $b$ in B. The purpose of this paper is to study the separability of $B[j]$. The separable extension of $B[j]$ over $B$ is characterized in terms of the trace $(=1+\sigma)$ of $\mathrm{B}$ over the subring of fixed elements under $\sigma$. Also, the characterization of a Galois extension of a commutative ring given by Parimala and Sridharan is improved.

KEY WORDS AND PHRASES. Quaternion Rings, Separable Algebras, and Galois Extensions.

AMS(MOS) SUBJECT CLASSIFICATION (1970) CODES. 16A16, 13A20, $13 B 05$. 


\section{INTRODUCTION.}

In [6], we studied the separable extension of group rings $R G$ and quaternion rings $R[i, j, k]$ over a ring $R$ with 1 . We have shown that $R[i, j, k]$ is a separable extension of $R$ if and only if 2 is a unit in $R$. Recently, S. Parimala and R. Sridharan ([5]) investigated another class of quaternion ring extensions $B[j]$ over a commutative ring $B$ with 1 and with an automorphism group $G(=\{\sigma\})$ of order 2 , where $B[j]$ is a free B-module with a basis $\{1, j\}, j^{2}=-1$, and $j b=\sigma(b) j$ for each $b$ in B. Their work is based on the following characterization of a Galois extension of a commutative ring ([5], Proposition 1.1): Let $A$ be the set of elements in $B$ fixed under 6 . Assume 2 is a unit in A. Then, B is Galois over $A$ if and only if $B \mathbb{A}_{A} B[j] \cong M_{2}(B)$, a matrix algebra over B of order 2, where the Galois extension is in the sense of Chase-Harrison-Rosenberg ([2]). The purpose of this paper is to study the separability of $B[j]$. Without the assumption that 2 is a unit in $A$, we shall characterize the separability of $B[j]$ in terms of the trace $(=1+6)$ of B over A. This shows the existence of a separable generalized quaternion ring extension $B[j]$ with 2 not a unit in $A$. When $\operatorname{Char}(A)=2$, we shall show that $B[j]$ is a separable extension over $B$ if and only if $B$ is Galois over A. Thus we can Improve the above theorem of Parimala and Sridharan. Then, the case in which 2 is a unit will be discussed, and several examples are constructed to illustrate our main results.

\section{PRELIMINARIES.}

Let us recall some basic definitions as given in [1], [2], [3], [4] and [6]. Let $B$ be a commutative ring containing a subring $A$ with the same identity 1. Then B is called a Galois extension over A ([2], or [3], Chapter 3) with a finite automorphism group G if (1) there exist 
elements $\left\{a_{i}, b_{i}\right.$ in $B / i=1,2, \ldots, n$ for some integer $\left.n\right\}$ such that $\sum a_{i} b_{i}=1$ and $\sum a_{i} \sigma\left(b_{i}\right)=0$ whenever $\sigma \neq 1$ in $G$, and (2) $A=\{b$ in $B$ $/ \sigma(b)=b$ for all $\sigma$ in $G\}$. The map $\Sigma \sigma$ is called the trace of $B$ over A denoted by $\mathrm{Tr}$. Let $S$ be a ring (not necessarily commutative) containing a subring $R$ with the same identity 1 . Then $S$ is called a separable extension of $R$ if there exist elements, $\left\{c_{i}, d_{i}\right.$ in $s / 1=1,2, \ldots, n$ for some integer $n\}$ such that (1) $a\left(\sum c_{i} d_{i}\right)=\left(\Sigma c_{i} d_{i}\right)$ a for all a in $S$ where is over $R$, and (2) $\sum c_{i} d_{i}=1$. Such an element $\sum c_{i} d_{i}$ is called a separable idempotent for $S$. When $R$ is contained in the center

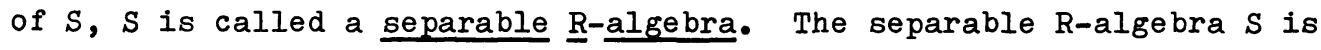
called an Azumaya R-algebra if $R$ is the center of $S$.

3. SEPARABLE QUATERNION ALGEBRAS.

Throughout, we assume that $B$ is a commutative ring with 1 , and $G$ $(=\{\sigma\})$ an automorphism group of order 2 of $B$, and that $B[j]$ is the generalized quaternion algebra over $A$, where $A$ is the subring of elements fixed under 6. Our main goal in the section is to study a separable extension $B[j]$ over $B$ without the assumption that 2 is a unit in $A$. We begin with a description of the set of separable idempotents for $B[j]$ (if there are any) over B. Clearly, $\{1 \otimes 1,10 j, j \otimes 1, j \otimes j\}$ is a basis for $B[j] \otimes_{B} B[j]$.

LEMMA 3.1. The element $x=a_{11}(1 \otimes 1)+a_{12}(1 \otimes j)+a_{21}(j \otimes 1)+a_{22}(j \otimes j)$ is a separable idempotent for $B[j]$ over $B$ if and only if (1) $a_{22}=-\sigma\left(a_{11}\right)$ such that $\operatorname{Tr}\left(a_{11}\right)=1$, and (2) $a_{21}=\sigma\left(a_{12}\right)$ such that $a_{12}((b-\sigma(b))=0$ for all $b$ in $B$ and $\operatorname{Tr}\left(a_{12}\right)=0$.

PROOF. Let $x$ be a separable idempotent for $B[j]$ over $B$. Then $x u=u x$ for each $u$ in $B[j]$. Hence $x j=j x$; that is,

$$
\sigma\left(a_{11}\right)(j 21)+\sigma\left(a_{12}\right)(j \otimes j)-\sigma\left(a_{21}\right)(121)-\sigma\left(a_{22}\right)(12 j)=
$$


$a_{11}(1 \otimes j)-a_{12}(1 \otimes 1)+a_{21}(j \otimes j)-a_{22}(j \otimes 1)$. Equating corresponding coefficients, we have $\sigma\left(a_{11}\right)=-a_{22}, a_{12}=6\left(a_{21}\right)$; that is, $a_{22}=-6\left(a_{11}\right)$ and $a_{21}=6\left(a_{12}\right)$ for $b^{2}=1$. Also, $b x=x b$ for $a l l b$ in $B$, so $b_{12}(b-\sigma(b))=0$. Thus $x=a_{11}(1 \otimes 1)+a_{12}(1 \otimes j)+\sigma\left(a_{12}\right)(j \otimes 1)-\sigma\left(a_{11}\right)(j \otimes j)$ with $a_{12}(b-b(b))=0$. Moreover, by the second condition of a separable idempotent, $a_{11}+\left(a_{12}+\sigma\left(a_{12}\right)\right) j+\sigma\left(a_{11}\right)=1$, so $\operatorname{Tr}\left(a_{11}\right)=1$ and $\operatorname{Tr}\left(a_{12}\right)=$ 0 . Conversely, it is straightforward to verify that any $x$ satisfying all equations as given is a separable idempotent.

THEOREM 3.2. $B[j]$ is a separable extension over $B$ if and only if there is an element $c$ in $B$ such that $\operatorname{Tr}(c)=1$.

PROOF. The necessity is a consequence of Lemma 3.1. For the sufficiency, if $\operatorname{Tr}(c)=1$, we take $a_{11}=c, a_{12}=a_{21}=0$. Then $a_{11}(1 \otimes 1)-$ $\sigma\left(a_{11}\right)(j \otimes j)$ is a separable idempotent for $B[j]$ by Lemma 3.1. Thus $B[j]$ is a separable extension over $B$.

Using Theorem 3.2, we can obtain a characterization of a separable extension $B[j]$ over $\bar{B}$ when $\operatorname{Char}(A)=2$.

THEOREM 3.3. Assume $\operatorname{Char}(A)=2$. Then, $B[j]$ is a separable extension over B if and only if B is a Galois extension over A.

PROOF. Let B be a Galois extension over A. Corollary 1.3 on P. 85 in [3] implies that $\operatorname{Tr}(c)=1$ for some $c$ in $B$. Thus $B[j]$ is a separable extension over B by Theorem 3.2. Conversely, by Theorem 3.2 again, there exists an $c$ in $B$ such that $\operatorname{Tr}(c)=1$, so $(c+b(c))=1$. By hypothesis, $\operatorname{Char}(A)=2, \sigma(c)=\sigma(-c)=-\sigma(c)$, so $c-\sigma(c)=1$. Hence the ideal generated by $\{(b-\sigma(b)) / b$ in $B\}=B$. This implies that $B$ is Galois over A by the statement 5 in Proposition 1.2 on P. 81 in [3]. Let us recall that the theorem of Parimala and Sridharan (Proposition 1.1 in [5]): Assume 2 is a unit in A. Then, B is Galois over A 
if and only if $B \otimes_{A} B[j] \cong M_{2}(B)$, a matrix algebra over $B$ of order 2 . We are going to improve it without the assumption that 2 is a unit in $A$. THEOREM 3.4. If $B$ is Galois over $A$, then $B{ }_{A} B[j] \cong M_{2}(B)$. PROOF. If $B$ is Galois over $A$, there exists an $c$ in $B$ such that $\operatorname{Tr}(c)=1$ ([3], Corollary 1.3, P. 85). Hence $B[j]$ is a separable extension over A by Theorem 3.2. But B is also a separable extension over A by Proposition 1.2 in [3], so the transitive property of separable extensions ([4], Proposition 2.5) implies that $B[j]$ is a separable A-algebra. Moreover, we claim that (1) $B[j]$ is an Azumaya algebra over A, and (2) B is a maximal commutative subalgebra of $B[j]$. The proof of these facts was given in [7]. For completeness, we give an outline here. For part (1), it suffices to show that $A$ is the center of $B[j]$. Clearly, $A$ is contained in the center. Now, let $b+b / j$ be in the center. Then $j\left(b+b^{\prime} j\right)=\left(b+b^{\prime} j\right) j$ and $\left.c\left(b+b^{\prime} j\right)=(b+b)^{\prime}\right) c$ for each $c$ in $B$. Equating coefficients of the basis $\{1, j\}$ in the above equations, we have that $\mathrm{b}$ is in $\mathrm{A}$ and $\mathrm{b}^{\prime}=0$ by Statement 5 in Proposition 1.2 on P. 81 in [3]. For part (2), to show that B is a maximal commutative subalgebra of $B[j]$ is to show that the commutant of $B$ in $B[j]$ is $B$. The computation is similar to part (1).

Moreover, noting that $B$ is separable over $A$, we then conclude that $B \otimes_{A}(B[j])^{O} \cong \operatorname{Hom}_{B}(B[j], B[j])$ by Theorem 5.5 on P. 65 in [3], and this implies that $B{ }_{A} B[j] \cong M_{2}(B)$, where $(B[j])^{\circ}$ is the opposite ring. In [7], the sufficiency of the Parimala and Sridharan theorem was shown by a different method from [5]. Now we slightly improve the statement without the assumption that 2 is a unit in $A$.

THEOREM 3.5. Let $B[j]$ be a separable extension over $B$. If $B{ }_{A} B[j] \cong M_{2}(B)$, then $B$ is Galois over $A$. 
PROOF. Since $B[j]$ is a separable extension over $B$, there exists an element $c$ in $B$ such that $\operatorname{Tr}(c)=1$ by Theorem 3.2. Hence the sequence $B \rightarrow A \rightarrow 0$ is exact under the trace map. But $A$ is projective over $A$, so the sequence splits, and then $A$ is an A-direct summand of $B$. By hypothesis, ${ }_{A} B[j] \cong M_{2}(B)$ which is an Azumaya $B$-algebra, so $B[j]$ is an Azumaya A-algebra ([3], Corollary 1.10, P. 45). Therefore B is Galois over A by using the same argument as given in [7].

In Theorem 3.5, the hypothesis that $B{ }_{A} B[j] \cong M_{2}(B)$ can be replaced by that $B{ }_{A} B[j]$ is an Azumaya $B$-algebra with the same proof.

4. SPECIAL SEPARABLE QUATERNION ALGEBRAS.

Theorem 3.5 tells us that $B[j]$ is an Azumaya A-algebra such that ${ }_{A} B[j] \cong M_{2}(B)$ when $B$ is Galois over $A$. In this section, we are going to discuss generalized quaternion algebras $B[j]$ in which 2 is a unit in $A$ when $B$ is projective and separable over A. With a similar argument as given in Lemma 3.1, we have

LEMMA 4.1. The element $a_{11}(1 \otimes 1)+a_{12}(1 \otimes j)+a_{21}(j \otimes 1)+a_{22}(j \otimes j)$ in $A[j]{ }_{A} A[j]$ is a separable idempotent for $A[j]$ if and only if (1) $a_{22}=$ $-a_{11}$ such that $2 a_{11}=1$, and (2) $a_{21}=a_{12}$ such that $2 a_{12}=0$. THEOREM 4.2. The A-algebra $A[j]$ is separable if and only if 2 is a unit in $\mathrm{A}$.

PROOF. The necessity is clear by Lemma 4.1; the sufficiency is immediate because $(1 / 2)(1 \otimes 1-j \otimes j)$ is a separable idempotent.

Now we give a characterization of $B[j]$ in which 2 is a unit when $B$ is projective and separable over A.

THEOREM 4.3. Let $B$ be separable and projective over A. Then, $B[j]$ is a separable extension over $B$ and projective over $A[j]$ as a bimodule if and only if 2 is a unit in $A$. 
PROOF. Let 2 be a unit in $A$ and let $c$ be $(1 / 2)$. Then $\operatorname{Tr}(c)=$ $1 / 2+1 / 2=1$, and hence $B[j]$ is separable over $B$ by Theorem 3.2. By hypothesis, B is projective over A, so $B[j]$ is left projective over $A$ (for $B[j]$ is left projective over $B$ ). Hence $B[j]$ is left projective over $A[j]$ ( $[3]$, Proposition 2.3, P. 48). We next claim that $B[j]$ is also right projective over $A[j]$. In fact, $\alpha: B{ }_{A} A[j] \rightarrow B[j]$ defined by $\alpha\left(b 1+b^{\prime} j\right)=b b^{\prime} j$ for $a l l b$ and $b^{\prime}$ in $B$ is an isomorphism as right $A[j]$-modules. But $B$ is projective over $A$, so $B{ }_{A} A[j]$ is right projective over $A[j]$. This proves that $B[j]$ is right projective over $A[j]$. Thus $B[j]{ }_{A}(B[j])^{\circ}$ is projective as $A[j]-A[j]-m o d u l e$. Since $B[j]$ is a direct summand of $B[j] \otimes_{A}(B[j])^{\circ}$ as a $B[j] \otimes_{A}(B[j])^{\circ}$-module (for $B[j]$ is separable over $A$ ), $B[j]$ is projective as a $A[j]-A[j]-m o-$ dule.

Conversely, to show that 2 is a unit in $A$, it suffices to show that $A[j]$ is a separable A-algebra by Theorem 4.2. Since $B[j]$ is a separable extension over $B, \operatorname{Tr}(c)=1$ for some $c$ in $B$ by Theorem 3.2. Hence $\operatorname{Tr}: \quad B \rightarrow A \rightarrow O$ is exact. We claim that $\operatorname{Tr}$ induces an exact sequence: $B[j] \rightarrow A[j] \rightarrow 0$ as $A[j]-A[j]$-modules. We define $\beta$ : $B[j] \rightarrow A[j] \rightarrow 0$ by $\beta\left(b+b^{\prime} j\right)=\operatorname{Tr}(b)+\operatorname{Tr}\left(b^{\prime}\right) j . \quad$ Clearly, $\beta$ is an additive group homomorphism. Moreover, for $a, a^{\prime}$ in $A,\left(b+b^{\prime} j\right)\left(a+a^{\prime} j\right)=$ $\left(b a-b^{\prime} a^{\prime}\right)+\left(b a^{\prime}+b^{\prime} a\right) j$, so $\beta\left(\left(b+b^{\prime} j\right)\left(a+a^{\prime} j\right)\right)=\operatorname{Tr}\left(b a-b^{\prime} a^{\prime}\right)+\operatorname{Tr}\left(b a^{\prime}+b^{\prime} a\right) j=$ $\left(a \operatorname{Tr}(b)-a^{\prime} \operatorname{Tr}\left(b^{\prime}\right)\right)+\left(a^{\prime} \operatorname{Tr}(b)+a \operatorname{Tr}\left(b^{\prime}\right)\right) j$. Also, $\beta\left(b+b^{\prime} j\right)\left(a+a^{\prime} j\right)=\left(\operatorname{Tr}(b)+\operatorname{Tr}\left(b^{\prime}\right) j\right)\left(a+a^{\prime} j\right)=\beta\left(\left(b+b^{\prime} j\right)\left(a+a^{\prime} j\right)\right)$. Thus $\boldsymbol{\beta}$ is a right $A[j]$-homomorphism. Similarly, by noting that $\operatorname{Tr}=1+\sigma$ and that $(\operatorname{Tr}) 6=\operatorname{Tr}=\sigma(\operatorname{Tr})$, it is straightforward to verify that $\beta$ is a left $A[j]$-homomorphism. But then $A[j]$ is $A[j]-A[j]$ projective such that $\boldsymbol{\beta}$ is onto (for $\operatorname{Tr}(c)=1$ in $A[j]$ ). This implies that the exact 
sequence $\beta: B[j] \rightarrow A[j] \rightarrow 0$ splits as $A[j]-A[j]$-modules. Thus $A[j]$ is an $A[j]$-direct summand of $B[j]$. Now by hypothesis, $B[j]$ is $A[j]$-projective, so $B[j] \otimes_{A}(B[j])^{\circ}$ is $A[j] \theta_{A} A[j]-p r o j e c t i v e$, where $(B[j])^{\circ}$ is the opposite algebra of $B[j]$. By hypothesis again, $B[j]$ is separable over $A$, so $B[j]$ is projective over $A[j] \mathbb{A}_{A} A[j]$. Therefore, the $A[j]-$ direct summand $A[j]$ of $B[j]$ is also projective over $A[j] \otimes_{A} A[j]$. This proves that $A[j]$ is separable over $A$, and so 2 is a unit in $A$ by Theorem 4.2 .

\section{EXAMPLES.}

This section includes several examples to illustrate our results.

(1) Let $\mathrm{Z}$ be the ring of integers, and $\mathrm{ZxZ}(=\mathrm{B})$ the ring of direct product of $\mathrm{Z}$ under the componentwise operations. Define $\sigma: \quad \mathrm{ZxZ} \rightarrow \mathrm{ZxZ}$ by $\sigma\left(a, a^{\prime}\right)=\left(a^{\prime}, a\right)$ for $a, a^{\prime}$ in $z$. Then $\sigma$ is an automorphism group of order 2 and $\{(a, a) / a$ in $Z\}(=A)$ is the subring of $Z x Z$ of the fixed elements under $\sigma$. Imbed $\mathrm{Z}$ in $\mathrm{ZxZ}$ by $\mathrm{a} \rightarrow(a, a)$. Then we have

(a) $\mathrm{ZxZ}$ is a free A-module with a basis $\{(1,0),(0,1)\}$.

(b) $\mathrm{ZxZ}$ is separable over $\mathrm{Z}$.

(c) $(\mathrm{ZxZ})[j]$ is a separable extension over $\mathrm{ZxZ}$ because $\operatorname{Tr}((1,0))=$ $(1,0)+(0,1)=(1,1)$ by Theorem $3 \cdot 2$.

(d) $Z[j]$ is not separable over $Z$ because 2 is not a unit in $Z$ by Theorem 4.2 .

(e) $(\mathrm{ZxZ})[j]$ is not projective over $z[j]$ because 2 is not a unit in $\mathrm{Z}$ by Theorem 4.3 .

(2) Let $Z_{(3)}$ be the local ring of $Z$ at the prime ideal (3). Replace $\mathrm{Z}$ by $\mathrm{Z}_{(3)}$ in Example (1). Then we have
(a) 2 is a unit in $Z_{(3)^{\circ}}$
(b) All properties (a), (b) and (c) in Example (1) hold. 


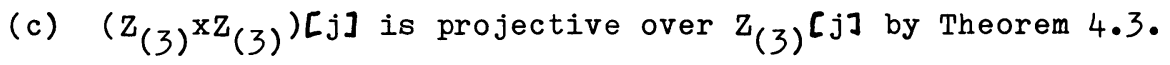

(3) $\mathrm{ZxZ}$ and $\mathrm{Z}_{(3)} \mathrm{xZ}_{(3)}$ in Example (1) and Example (2) are Galois over $\mathrm{Z}$ and $\mathrm{Z}_{(3)}$ respectively by using Proposition 1.2 on P. 64 in [3], Since $\operatorname{Tr}((3,-2))=(3,-2)+(-2,3)=(1,1)$ which is not in any maximal ideal of $\mathrm{ZxZ}$ or $\mathrm{Z}_{(3)} \mathrm{xZ}(3)$. Thus $(\mathrm{ZxZ}) \mathrm{Q}_{\mathrm{Z}}(\mathrm{ZxZ})[j] \cong \mathrm{M}_{2}(\mathrm{ZxZ})$ and

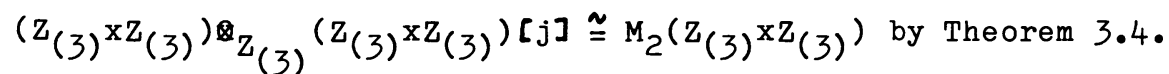

(4) Let $i$ be the usual imaginary unit. Then $z[i]$ is not separable over $z$. $z[i]$ has an automorphism group $\{\sigma: \sigma(a+b i)=a-b i$ for $a, b$ in $z\}$ such that $\sigma^{2}=1$ and $z$ is the fixed ring of 6 . Also, (a) (z[i]) $[j]$ is not separable over $Z[i]$, and (b) $Z[i]$ is not Galois over $Z$.

\section{REFERENCES}

1. Auslander, M. and 0. Goldman. The Brauer Group of a Commutative Ring, Trans. Amer. Math. Soc. 97 (1960) 367-409.

2. Chase, S., D. Harrison and A. Rosenberg. Galois Theory and Galois Cohomology of Commutative Rings, Mem. Amer. Math. Soc. 22 (1965).

3. DeMeyer, F. and E. Ingraham. Separable Algebras over Commutative Rings, Springer-Verlag, Berlin-Heidelberg-New York, 181, 1971.

4. Hirata, K. and K. Sugano. On Semisimple Extensions and Separable Extensions over Non-Commutative Rings, J. Math. Soc. Japan 18 (1966) 360-373.

5. Parimala, S. and R. Sridharan. Projective Modules over Quaternion Algebras, J. Pure and Applied Alg. 9 (1977) 181-193.

6. Szeto, G. On Separable Extensions of Group Rings and Quaternion Rings, International J. Math. and Math. Sciences, 1 (1978) 433-438.

7. Szeto, G. On Galois Extensions of Commutative Rings, Proc. Conf. on Ring Theory, Antwerp, Belgium, Marcel Dekker Publi., 1979. 


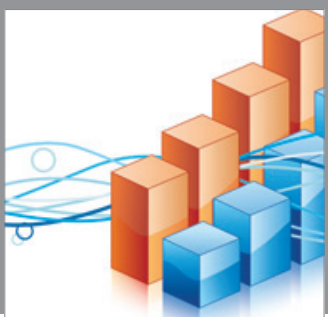

Advances in

Operations Research

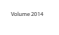

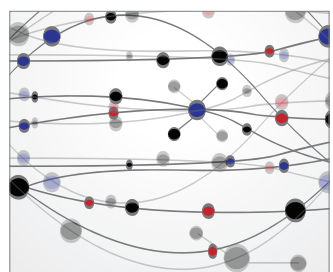

\section{The Scientific} World Journal
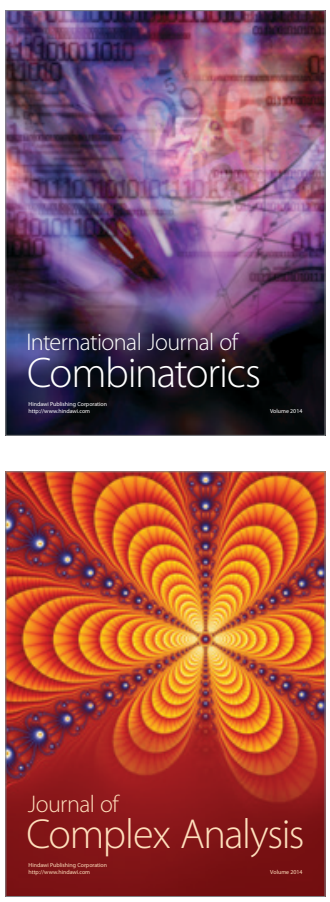

International Journal of

Mathematics and

Mathematical

Sciences
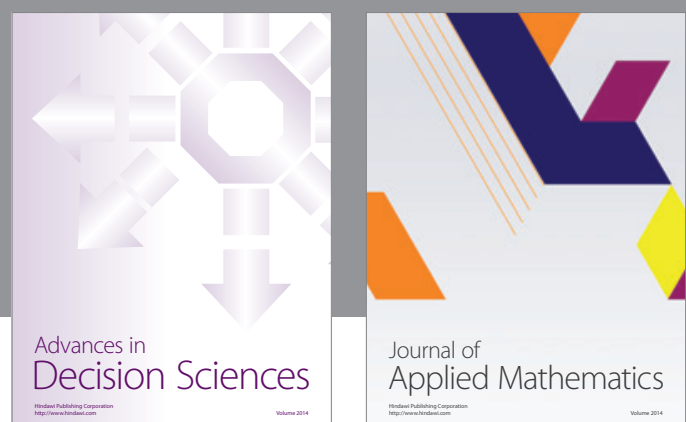

Journal of

Applied Mathematics
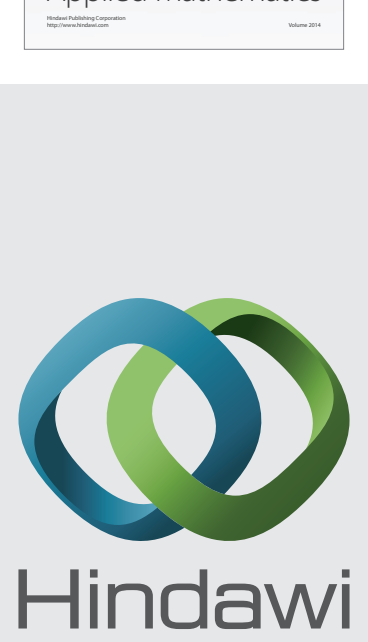

Submit your manuscripts at http://www.hindawi.com
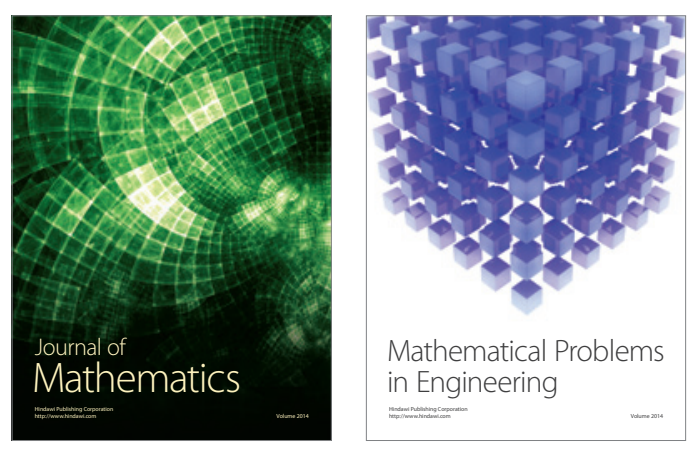

Mathematical Problems in Engineering
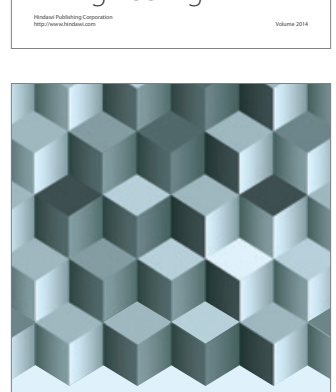

Journal of

Function Spaces
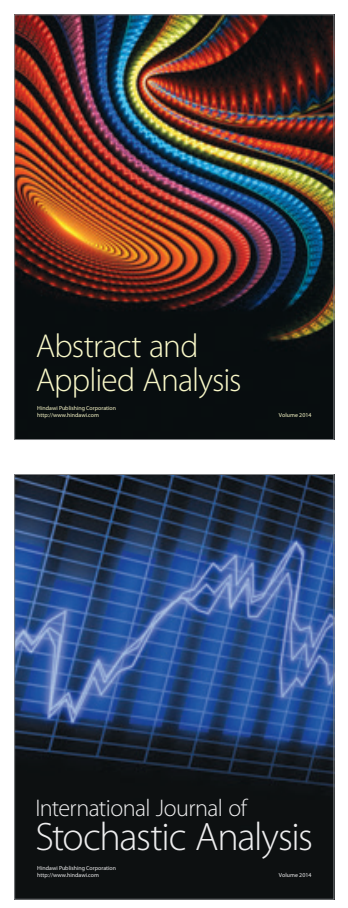

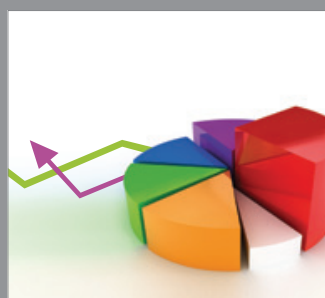

ournal of

Probability and Statistics

Promensencen
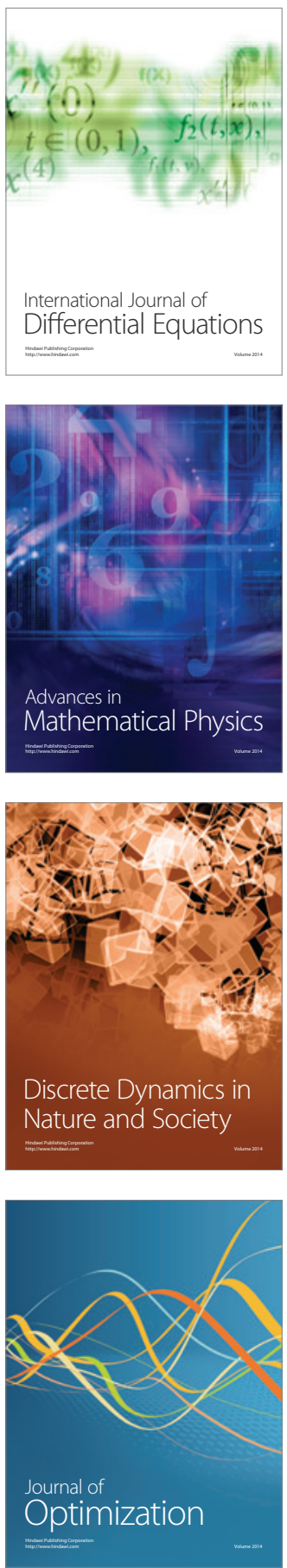